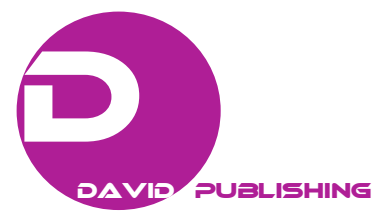

\title{
Morphological Parameters of Bladder Rats with Infravesical Obstruction with the Introduction of Biologically Active Compositions
}

\author{
Vyacheslav Yu. Globa, Galyna A. Bozhok and Evgeniy I. Legach \\ Department of Cryoendocrinology, Institute for Problems of Cryobiology and Cryomedicine of the National Academy of Sciences of \\ Ukraine, Kharkiv 61016, Ukraine
}

\begin{abstract}
The morphological features of the urinary bladder (UB) after administration of biologically active compositions (BACs) containing neurotrophic factors (NFs) in female rats with infravesical obstruction (IVO) were studied. IVO was modeled by the ligature method. A cryo extract of the spinal ganglia (SG), conditioned media from cultures of native and cryopreserved mantle gliocytes (MG), and the drug Cortexin were used as the BACs. The BACs were injected intraperitoneally to the animals for 10 days. The morphometric parameters of the UB were determined by studying all its shells and their structure. The BACs obtained from the SG participated in the remodeling of the UB wall, which was changed during the IVO. Exogenous NFs insignificantly affected the hyperplasia of the transitional epithelium, stimulated regression and progression of the enlarged muscle layer of the detrusor, increased the proliferation of connective tissue, and increased the wall thickness of the UB. Proceeding from the above, NFs reduced the indices of the ratio of the muscle layer/wall of the UB, mediated by IVO.
\end{abstract}

Key words: Bladder morphology, infravesical obstruction, spinal ganglion, cryo extract, cryopreservation.

\section{Introduction}

Currently, urination disorders caused by infravesical obstruction (IVO) are observed in $21.5 \%$ of the world's population. IVO causes multiple changes in the smooth muscles of the UB, transitional epithelium, nerves and vasculature. This leads to progressive hypertrophy and impaired UB contractility [1].

Today, methods of regenerative medicine are relevant, restoring disturbed physiological processes and affecting the survival, differentiation and growth of cells of various tissues of the urinary system [2]. For these purposes, neurotrophic drugs are used, one of which is cortexin, which have a predominant effect on the nervous system. Correction of the main pathogenetic mechanisms of IVO, such as hypertrophy and impaired UB contractility, requires a combination of therapeutic and surgical approaches,

Corresponding author: Vyacheslav Yuryovich Globa, graduate student, research field: cryomedicine, urology. often with unsatisfactory results [3].

There are works showing the participation of NFs in the remodeling of the UB wall after IVO [4]. This allows the use of BACs (extracts, conditioned media, lysates) to influence the morphological and functional properties of UB in health and disease.

NFs are localized in tissues with high proliferative activity, especially in glial cells of the peripheral and central nervous systems [5]. Spinal ganglia (SG) and cell cultures from them are a potential source of NFs [6].

Currently, there are few studies that study the possibility of using BACs obtained from SG on the morphological indicators of UB in IVO.

\section{Materials and Methods}

\subsection{Study Design}

White outbred rats were used for research: females weighing 250-320 g and 6 months of age. All manipulations with animals were carried out in 
accordance with the Law of Ukraine "On the Protection of Animals from Cruelty" (No. 3447-IV dated 02.21.2006) in compliance with the provisions of the "European Convention for the Protection of Vertebrate Animals Used for Experimental or Other Scientific Purposes" (Strasbourg, 1986).

The cryo extract was obtained from the SG of sexually mature rats using an original method [7]. After that, the cryo extract was passed through a filter with a pore diameter of $0.22 \mu \mathrm{m}$ (TPP, Switzerland) for sterilization and packed in $1.5 \mathrm{~mL}$ in microtubes Eppendorf (Greiner Bio-One GmbH, Germany), followed by freezing in a refrigerator at $-18{ }^{\circ} \mathrm{C}$. The protein concentration in the SG cryo extract was determined by the Bradford method and amounted to $0.1 \mathrm{mg} / \mathrm{mL}$ [8]. Before use, the cryo extract was warmed in a water bath $\left(42{ }^{\circ} \mathrm{C}\right)$ with continuous shaking for 1-2 $\mathrm{min}$ and centrifuged in a mini-centrifuge $(2,000 \mathrm{~g})$ for $10 \mathrm{~min}$.

The culture of mantle gliocytes (MG) was obtained from the SG of neonatal piglets by an enzymatic method [9]. The initial seeding concentration of cells in both cases was $6 \times 10^{4}$ cells $/ \mathrm{cm}^{2}$. On the $21 \mathrm{st}$ day of cultivation, the medium was collected from all Petri dishes, and combined; an aliquot was taken and used in further experiments.

Part of the SG cell cultures after cultivation for 6 days was detached from the substrate with $0.25 \%$ trypsin-EDTA solution with Hanks salts (PanEco, Russia) and cryopreserved as a suspension in cryoprotective medium with $10 \%$ dimethyl sulfoxide (DMSO) with a cooling rate of $1{ }^{\circ} \mathrm{C} / \mathrm{min}$ to $-40{ }^{\circ} \mathrm{C}$ and further immersion in liquid nitrogen. The cells were kept at $-196{ }^{\circ} \mathrm{C}$ for 1 month, after which they were thawed, washed from DMSO, and cultured for 20 days. On day 21, the culture medium was collected from all samples, combined; an aliquot was taken and used in further experiments.

IVO was modeled by the ligature method [10].

After 1.5 months, the ligature was removed and the next day the animals were started to become injected the BACs intraperitoneally for 10 days. Cryo extract of $\mathrm{SG}$, conditioned medium obtained from native and cryopreserved MG cultures were injected with 0.6 $\mathrm{mL} / \mathrm{kg}$ of body weight. Cortexin (Geropharm, Russia) was administered at a dose of $1.0 \mathrm{~mL} / \mathrm{kg}$ of body weight [11]. The animals were taken out of the experiment by decapitation under anesthesia on the 56th day from the start of IVO modeling.

The animals were divided into groups: 1-control (C); 2-the introduction of neurotrophic drug Cortexin (NDC); 3-introduction of conditioned medium from the culture of native MG (CNMG); 4-introduction of conditioned medium from the culture of cryopreserved MG (CCMG); 5-introduction of SG cryo extract (SGC); 6-without treatment (U).

\subsection{Morphologic Analysis of the Urinary Bladder}

For morphological assessment, UB fragments were subjected to histological tracing, stained with hematoxylin and eosin according to the standard technique [12]. Microphotography was carried out using an AmScope light-optical microscope, model XYL-403 (China) with a digital camera. The morphometric analysis of micrographs of serial sections of the UB was performed using the AxioVision Rel 4.7 image processing software (Carl Zeiss, Germany). The medium height of the transitional epithelium, the thickness of the muscle layer, the total wall thickness of the UB, the index of the ratio of the muscle layer/wall of the UB on histological preparations were measured on cross sections. Ten (10) measurements were performed on 1 slice, 5-6 slices were counted for each sample.

\subsection{Statistical Analysis}

The results were statistically processed using Excel (Microsoft, USA) and Statistica 10 (Statsoft, USA). Quantitative data were presented as median (Me) and quartiles (Q1; Q3) and were assessed using nonparametric Kruskal-Wallis and Mann-Whitney tests. Differences were considered statistically significant at $p<0.05$. 


\section{Results}

\subsection{General Histological Characteristic of the Urinary Bladder}

In the morphological study of the UB of control rats, the mucous membrane was covered with transitional epithelium, which was located on a loose lamina of its own. The muscular layer had an ordered structure in the form of several layers of smooth muscles with a thin layer of connective tissue between them. The serous membrane was represented by a thin layer of connective tissue outside the organ in Fig. 1A.

In the UB of animals treated with Cortexin (NDC), the transitional epithelium was moderately hypoplastic, there was a slight proliferation of the connective tissue of the lamina propria. The muscle layers of the lamina propria and detrusor retained a normal structure, but in places the muscles were hypertrophied. The spaces of connective tissue between the muscle layers expanded. The serous membrane was moderately thickened in Fig. 1B.

In animals of the 3rd group (CNMG), the surface of the mucous membrane of the UB was covered with hyperplastic epithelium. The lamina propria looked sharply thickened; its muscle layer was atrophied in places. The detrusor muscles lost their structure, consisted of groups of thickened muscle fibers with a small amount of connective tissue between them. The serous membrane was sharply thickened in Fig. 1C.

In the 4th group of rats (CCMG), the transitional epithelium of the UB looked sharply hyperplastic, but in some places there were areas of atrophy. The muscle layer of the lamina propria and detrusor muscles were sharply hypertrophied, with a moderate content of connective tissue between the layers. The serous membrane was sharply thickened in Fig. 1D.

In rats treated with a cryo extract of SG (SGC), foci of normal and hypoplastic transitional epithelium alternated in the mucous membrane of the UB. The lamina propria was almost replaced by connective tissue, and its muscle elements were partially atrophied. The muscular layer was moderately hypertrophied and consisted of regularly spaced smooth muscle bundles with a small amount of connective tissue. The serous membrane was slightly thickened in Fig. 1E.

Microscopic assessment of UB in rats of the 6th group (U) showed that the transitional epithelium was sharply hypoplastic and atrophied in places, and there were areas of its complete absence. The lamina propria was thinned, completely replaced by connective tissue, with the presence of hypertrophied muscles in the form of separate foci. The muscle layers lost their structural orientation, were represented by sharply hypertrophied bundles of muscle fibers, between which the connective tissue layer was reduced. The serous membrane looked sharply thickened in Fig. 1F.

\subsection{Morphometric Characteristic of the Urinary Bladder}

Measurement of the average height of the transitional epithelium of the UB revealed a decrease in the 5th and 6th groups by $24.3 \%$ and $20.5 \%$ (Fig. $2 \mathrm{~A})$, respectively, compared with the control. But the difference in this indicator between the 6th group (U) and the animals that received the BACs was statistically insignificant (Fig. 2A, $p>0.05$ ), except for the 3rd group. The thickness of the muscle layer of the UB increased in animals of the 4th, 5th and 6th groups by $42.07 \%, 18.5 \%$ and $37.26 \%$ (Fig. 2B), respectively, compared with the control. At the same time, the degree of muscle hypertrophy in the 2nd and 3 rd groups was minimal and approached the control values, but statistically significantly differed from the indicators of the 6th group.

Morphometric analysis revealed an increase in the total wall thickness of the UB in all groups, except for the 3rd (CNMG), by 21.95\%, 39.99\%, 32.5\% and $32.74 \%$ (Fig. 2C), respectively, compared with control. It is interesting that the indicators of the 1st (C) and 3rd groups were similar, but different from the values 
of the 6th group (U).

When examining the index of the ratio of the muscle layer/wall of the UB, a decrease of 1.2 times was found in the 2nd (NDC) and 3rd (CNMG) groups, compared with the rest of the groups (Fig. 2D). The highest index values were in the 6th group (U).
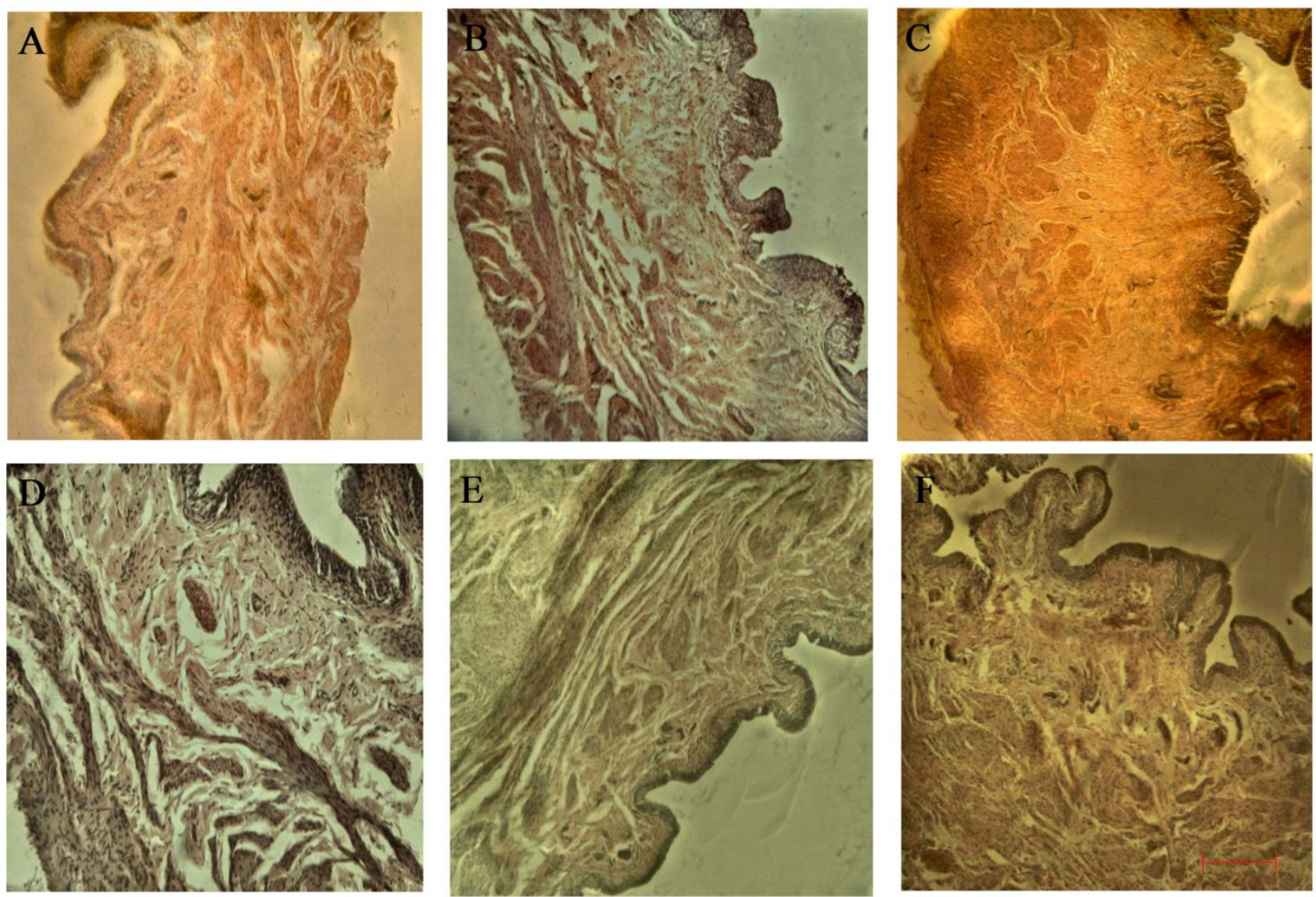

Fig. 1 Histopathology examination of different studied groups ( $\mathrm{H}$ and $\mathrm{E}$ stain, observed at $10 \times$ magnification; scale bar, 50 $\mu \mathrm{m})$. (A): control; (B): neurotrophic drag cortexin; (C): conditioned culture medium of native MG; (D): conditioned culture medium of cryopreserved MG; $(\mathrm{E})$ : cryo extract of SG; (F): without treatment. 

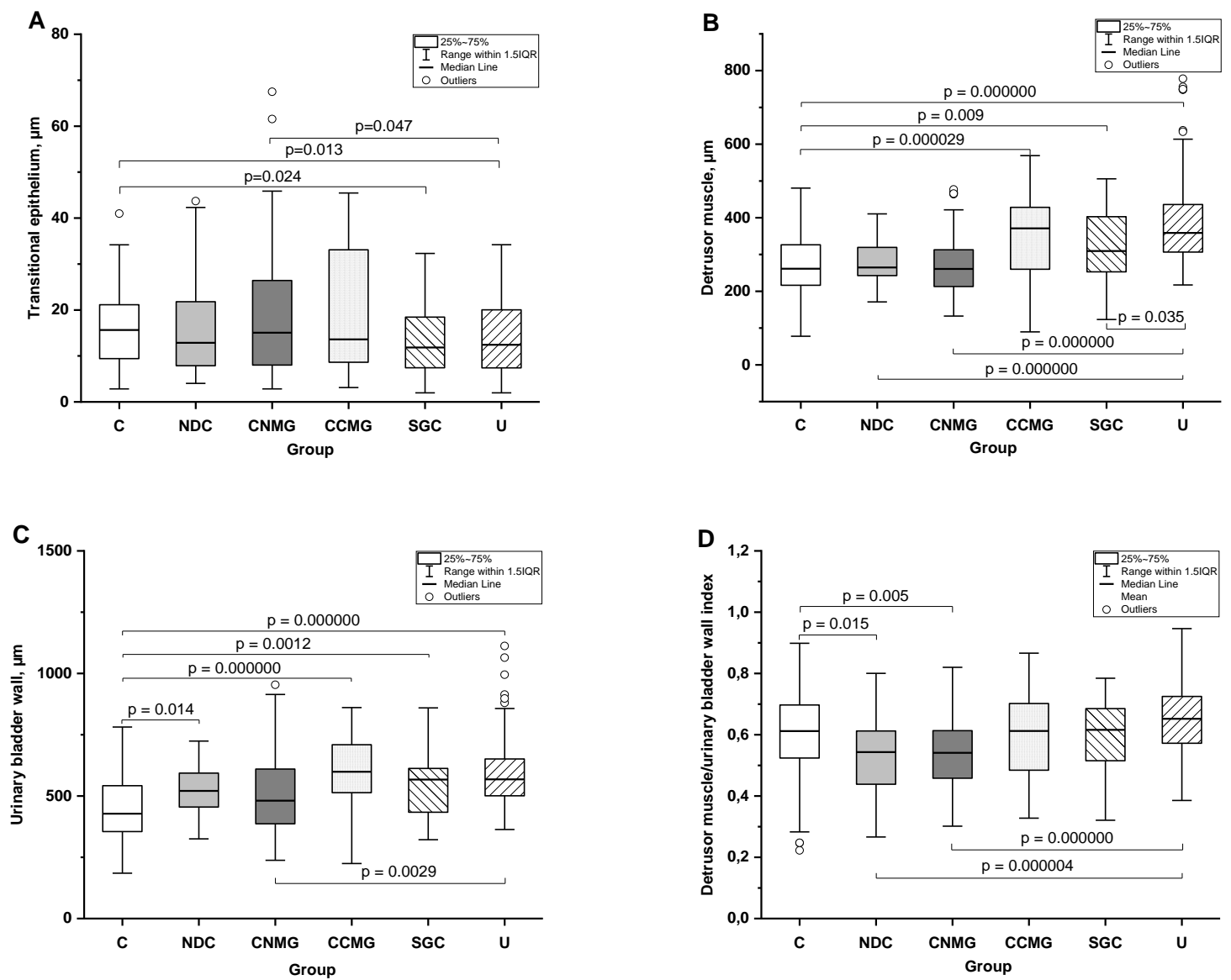

Fig. 2 The average height of the transitional epithelium (A), the thickness of the muscle layer (B), the total wall thickness of the UB, (C) and index of the ratio of the muscle layer/UB wall, (D) of different studied groups.

\section{Discussion}

We searched for works that studied the effect of BACs obtained from SG on the morphofunctional indicators of UB in experimental animals and humans after IVO. The obtained results indicate a small number of studies on this topic $[4,13]$. Basically, in the majority of scientific works, stem cells were used for the purpose of tissue engineering [2, 14], the effect of increased contractility of UB and endogenous NFs, growth factors on the morphology and function of the urinary tract [12, 15-18], the effect of high hydrostatic pressure on different cell cultures [19], the role of NFs in neurodegenerative diseases $[5,20]$. The increased interest in this topic was caused by the results we obtained in previous works. We investigated the contractility of the UB and the biochemical parameters of the blood of rats after IVO due to the use of BACs obtained from SG. In animals that were injected with a cryo extract of SG and conditioned medium obtained from a culture of cryopreserved MG, the most pronounced increase in body weight, the smallest indicators of UB weight and mass coefficient were found, and an increase in contractile activity and maximum amplitude of bladder contractions in response to various stimuli was noted [21, 22]. The results of the current study were probably obtained by us due to the fact that NFs mediate hypertrophy of smooth muscle cells, hyperplasia of transitional cell epithelium and connective tissue [4, 23]. At the same time, different types of growth factors and NFs can be both mitogens and growth inhibitors, depending on the type of cells and the state of cell differentiation, and can also express each other in UB tissues [12, 13, 
18]. Also indicative are the obtained indices of the muscle layer/wall of the UB, which have proven effective in detecting hypertrophy of the bladder wall due to IVO [24]. Based on the foregoing, it can be assumed that, in the future, it is advisable to use NFs obtained from SG and from the MG culture after freezing-warming, due to their wide spectrum of action on the morphological and functional properties of UB. This is possible due to the fact that after cryopreservation the ratio of morphological cell types changes, that is, their production of NFs [25]. At the same time, the use of neurotrophic drugs such as cortexin is less justified due to their predominant effect mainly on the nervous system. Our studies have confirmed the versatile effects of NFs along with increased hydrostatic pressure due to IVO on the growth, differentiation and apoptosis of smooth muscle cells of transitional cell epithelium and UB detrusor $[14,18,19]$.

\section{Conclusions}

A pronounced effect of exogenous NFs on transitional epithelial hyperplasia was not observed in experimental IVO (reduction in the height of the transitional epithelium only in the SGC group by $24.3 \%$ ). The introduction of native BACs stimulated regression of the enlarged UB detrusor to the control values, the thickness of which usually increases with IVO. At the same time, the use of NFs obtained by freezing-warming showed a positive effect on detrusor hypertrophy, presumably due to additional growth factors, increasing it by $18.5 \%$ (SGC) and $42.1 \%$ (CCMG). The use of BACs increased the proliferation of connective tissue and increased the wall thickness of the UB by $22 \%, 40 \%, 32.5 \%$ and $33 \%$. BACs obtained from $\mathrm{SG}$ reduce the indices of the ratio of the muscle layer/wall of the UB, mediated by IVO, by an average of $11.5 \%$.

All of the above conclusions allow us to speak about the positive participation of exogenous NFs in the remodeling of the UB wall changed during IVO.
This will make it possible not only to use BACs obtained from SG for therapeutic purposes, but also to develop methods for the prevention and prediction of many pathologies.

\section{References}

[1] Wein, A. J., Andersson, K. E., Drake, M. J., and Dmochowski, R. R. 2014. Bladder Dysfunction in the Adult: The Basis for Clinical Management. Current Clinical Urology. New York: Springer Science + Business Media.

[2] Imamura, T., Ishizuka, O., and Nishizawa, O. 2013. "Bone Marrow-Derived Cells Regenerate Structural and Functional Lower Urinary Tracts.” IntechOpen. Accessed May 22nd, 2013. https://www.intechopen.com/books/-regenerative-medici ne-and-tissue-engineering/bone-marrow-derived-cells-reg enerate-structural-and-functional-lower-urinary-tracts.

[3] Kim, S. J., Bae, W. J., and Kim, S. W. 2019. "Influence of Solifenacin on the Improvement of Storage Symptoms in the Early Period after Photoselective Vaporization of the Prostate." Investigative and Clinical Urology 60 (6): 480-7.

[4] Arms, L., and Vizzard, M. A. 2011. "Neuropeptides in Lower Urinary Tract Function." Handbook of Experimental Pharmacology 202: 395-423.

[5] Skaper, S. D. 2012. "The Neurotrophin Family of Neurotrophic Factors: An Overview." Methods in Molecular Biology 846: 1-12.

[6] Haberberger, R. V., Barry, C., Dominguez, N., and Matusica, D. 2019. "Human Dorsal Root Ganglia." Frontiers in Cellular Neuroscience 13: 271.

[7] Galchenko, S. Y., Shkodovska, N. Y., Sandomirsky, B. P., and Grischenko, V. I. 2004. "Method of Obtaining of Xenogeneic Organ Extracts." UA Patent 64381 A, IPC7 A61K35/12, №2003054649, filed May 22, 2003, and issued February 16, 2004. (in Ukrainian)

[8] Bradford, M. M. 1976. "A Rapid and Sensitive Method for the Quantitation of Microgram Quantities of Protein Utilizing the Principle of Protein-Dye Binding." Analytical Biochemistry 72 (1-2): 248-54.

[9] Ali, S. G., Sidorenko, O. S., and Bozhok, G. A. 2018. "Influence of Nutrient Medium Composition on the Morphological Characteristics of Culture of Dorsal Root Ganglion Cells of Neonatal Piglets.” The Journal of V. N. Karazin Kharkiv National University. Series «Biology» 30 (30): 49-59. (in Ukrainian)

[10] Zhang, N. Z., Ma, L., Zhang, J. B., and Chen, J. 2014. "Improved Model for the Establishment and Evaluation of Detrusor Overactivity in Female Wistar Rats." 
Biologically Active Compositions

International Brazilian Journal of Urology 40 (3): 414-22.

[11] Shavlovskaya, O. A. 2016. "Clinical Efficacy of Neuropeptides in Cerebrovascular Pathology." $S$. $S$. Korsakov Journal of Neurology and Psychiatry 116 (8): 88-93. (in Russian)

[12] Ku, J. H., Kim, Y., Moon, K. C., Kim, Y. S., Kim, M.-S., Kim, H. H., and Paick, J.-S. 2006. "In Vivo Hepatocyte Growth Factor Gene Transfer to Bladder Smooth Muscle after Bladder Outlet Obstruction in the Rat: A Morphometric Analysis." The Journal of Urology 176 (3): 1230-5.

[13] Liu, W., Li, Y., Cunha, S., Hayward, G., and Baskin, L. 2000. "Diffusable Growth Factors Induce Bladder Smooth Muscle Differentiation." In Vitro Cellular and Developmental Biology-Animal 36 (7): 476-84.

[14] Adamowicz, J., Juszczak, K., Bajek, A., Tworkiewicz, J., Nowacki, M., Marszalek, A., Thor, P. J., Chlosta, P., and Drewa, T. 2012. "Morphological and Urodynamic Evaluation of Urinary Bladder Wall Regeneration: Muscles Guarantee Contraction but Not Proper Function-A Rat Model Research Study." Transplantation Proceedings 44 (5): 1429-34.

[15] Aikawa, K., Ishibashi, K., Sakai, T., Takahashi, N., Ogawa, S., Kawashima, Y., and Yamaguchi, O. 2012. "Effect of the Renin-Angiotensin System on the Obstructed Bladder." LUTS: Lower Urinary Tract Symptoms 4: 81-6.

[16] Chul Kim, J., Il Seo, S., Hyun Park, Y., and Kon Hwang, T. A. E. 2001. "Changes in Detrusor and Urinary Growth Factors according to Detrusor Function after Partial Bladder Outlet Obstruction in the Rat." Urology 57 (2): 371-5.

[17] Sellers, D., Chess-Williams, R., and Michel, M. C. 2018. "Modulation of Lower Urinary Tract Smooth Muscle Contraction and Relaxation by the Urothelium." Naunyn-Schmiedeberg's Archives of Pharmacology 391 (7): 675-94.

[18] Sidler, M., Aitken, K., Jiang, J., Bijos, D., Belik, J., and
Bägli, D. J. 2017. "Finding NeMO-Nerve-sparing Mid-urethral Obstruction: A Pathophysiologically Accurate Model of Rodent Partial Bladder Outlet Obstruction." Urology 105: 208.e1-208.e9.

[19] Liu, S., Tao, R., Wang, M., Tian, J., Genin, G. M., Lu, T. J., and Xu, F. 2019. "Regulation of Cell Behavior by Hydrostatic Pressure.” Applied Mechanics Reviews 71 (4): 0408031-04080313.

[20] Gill, B. C., Balog, B. M., Dissaranan, C., Jiang, H.-H., Steward, J. B., Lin, D. L., and Damaser, M. S. 2012 "Neurotrophin Therapy Improves Recovery of the Neuromuscular Continence Mechanism Following Simulated Birth Injury in Rats." Neurourology and Urodynamics 32 (1): 82-7.

[21] Globa, V. Y., Samburg, Y. Y., Bozhok, G. A., and Legach E. I. 2020. "Biochemical Blood Parameters of Rats with Infravesical Obstruction with the Introduction of Biologically Active Compositions Containing Neurotrophic Factors." Emergency Medicine 16 (4): 115-20. (in Ukrainian)

[22] Globa, V. Y., Bondarenko, T. P., Bozhok, G. A., Samburg, Y. Y., and Legach, E. I. 2020. "Biologically Active Compositions Containing Neurotrophic Factors Change the Contractile Activity of Detrusor of Rats with Infravesical Obstruction." Problems of Cryobiology and Cryomedicine 30 (2): 188-98.

[23] Cruz, C. D. 2014. "Neurotrophins in Bladder Function: What Do We Know and Where Do We Go from Here?" Neurourology and Urodynamics 33 (1): 39-45.

[24] Abdel-Aal, A., El-Karamany, T., Al-Adl, A. M., Abdel-Wahab, O., and Farouk, H. 2011. "Assessment of Noninvasive Predictors of Bladder Outlet Obstruction and Acute Urinary Retention Secondary to Benign Prostatic Enlargement." Arab Journal of Urology 9 (3): 209-14.

[25] Ali, S., and Bozhok, G. 2019. "Effect of Cryopreservation on Different Passages of Porcine Dorsal Root Ganglion Cell Culture." Recent Advances in Biology and Medicine 5: 1-5. 